\title{
Influência do peso corporal e do índice de massa corporal no teste de sobrecarga hídrica
}

\author{
The influence of body weight and body \\ mass index on the water-drinking test
}

Sergio Henrique Sampaio Meirelles', Kahlil Ruas Ribeiro Mendes², Riani Morelo Álvares³, Ana Carolina de Magalhães Villela ${ }^{4}$, Diogo Arruda Câmara Pereira de Lucena ${ }^{5}$, Riuitiro Yamane ${ }^{6}$

\section{ResUmo}

Objetivo: Avaliar a influência do peso corporal e do índice de massa corporal (IMC) nos picos e na flutuação da pressão intra-ocular (PIO) no teste de sobrecarga hídrica (TSH) em pacientes com glaucoma primário de ângulo aberto (GPAA), glaucoma de pressão normal (GPN) e olhos normais (ON). Métodos: Estudo transversal em que foram avaliados 32 olhos de 32 pacientes com GPAA, 30 olhos de 30 pacientes com GPN e 20 olhos de 20 ON. Nenhum dos pacientes glaucomatosos havia iniciado o tratamento da doença. O TSH foi realizado às $17 \mathrm{~h}$, sendo avaliada a correlação entre o peso corporal e o IMC e os picos e a flutuação da PIO durante o TSH. Os resultados foram analisados através dos testes de Anova-fator único com teste de Bonferroni e correlação linear de Pearson. Foi considerado significativo valor de p menor que 0,05. Resultados: Não houve diferença significativa na idade $(\mathrm{p}=0,13)$, no peso corporal $(\mathrm{p}=0,13)$ e no $\operatorname{IMC}(\mathrm{p}=0,83)$ entre os três grupos. Foi encontrada correlação estatisticamente significativa no grupo GPAA entre o peso corporal e a flutuação da PIO no TSH $(\mathrm{p}=0,02 ; \mathrm{r}=-0,42)$ e entre o IMC e a flutuação da PIO no TSH $(\mathrm{p}=0,02 ; \mathrm{r}=-0,41)$. Não houve correlação significativa entre o peso corporal e o IMC e os picos de PIO nos três grupos e entre o peso corporal e o IMC e a flutuação da PIO nos grupos GPN e ON.. Conclusão: Os resultados do presente estudo sugerem que o peso corporal e o IMC devem ser considerados ao se avaliar os resultados do TSH em portadores de GPAA e que pacientes portadores de GPAA com maior IMC e maior peso corporal podem apresentar menor flutuação da PIO no TSH.

Descritores: Glaucoma de ângulo aberto/diagnóstico; Pressão intra-ocular;Técnicas de diagnóstico oftalmológico;Tonometria ocular/métodos; Peso corporal; Índice de massa corporal; Estudos de casos e controle

\footnotetext{
${ }^{1}$ Doutor, Professor da Universidade Gama Filho, Chefe do Setor de Glaucoma do Hospital Municipal da Piedade - Rio de Janeiro (RJ), Brasil;

${ }^{2}$ Residente do Hospital Municipal da Piedade - Rio de Janeiro (RJ), Brasil;

${ }^{3}$ Residente do Hospital Municipal da Piedade - Rio de Janeiro (RJ), Brasil;

${ }^{4}$ Mestre em Oftalmologia pela Universidade Federal do Rio de Janeiro - UFRJ

${ }^{5}$ Médico do Hospital da Lagoa - Rio de Janeiro (RJ), Brasil;

${ }^{6}$ Livre-docente, Professor Titular de Oftalmologia da Universidade Estadual do Rio de Janeiro (RJ), Brasil.
}

Trabalho realizado no Hospital Municipal Piedade - Rio de Janeiro (RJ), Brasil e Universidade Gama Filho Rio de Janeiro (RJ), Brasil.

Recebido para publicação em: 5/3/2008 - Aceito para publicação em 11/6/2008 


\section{INTRODUÇÃO}

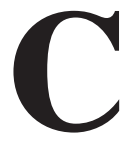

onsidera-se o aumento da pressão intra-ocular (PIO) o maior fator de risco para o desenvolvimento do glaucoma. Desta forma, muitas pesquisas têm sido realizadas com o objetivo de determinar os melhores métodos de diagnóstico e acompanhamento desta doença ${ }^{(1)}$.

O teste de sobrecarga hídrica (TSH) foi inicialmente descrito por Schmidt ${ }^{1}$ em 1928, que ao observar os estudos anteriores de Marx sobre as alterações sanguíneas produzidas pela ingestão de 1 litro de água, notou haver um aumento da PIO em pacientes glaucomatosos ${ }^{(2)}$.

Embora a utilização clássica do TSH para o diagnóstico do glaucoma ${ }^{(3)}$ tenha entrado em desuso para esta finalidade, pelo alto número de falso-positivos ${ }^{(4)}$, ele foi considerado como capaz de detectar o pico de Pio diário de pacientes glaucomatosos ou com suspeita de glaucoma ${ }^{(5)}$. Outro estudo ${ }^{(6)}$, ao correlacionar picos de Pio da curva diária de PIO (CDPIO), com o TSH, também encontrou valores semelhantes pelos dois métodos.

A variação da PIO durante o TSH foi considerada ainda como fator de risco para a progressão de defeitos de campo visual no glaucoma ${ }^{(7)}$ ou como capaz de avaliar o sucesso da trabeculectomia ${ }^{(8.9)}$ ou a eficácia do tratamento clínico do glaucoma ${ }^{(10)}$.

O mecanismo de elevação da PIO pelo TSH seria devido ao aumento da pressão venosa episcleral ${ }^{(11)}$. No entanto, pouco se sabe sobre a relação entre a quantidade de água ingerida de acordo com a massa corporal e a resposta do TSH. Um estudo considerou que o peso do indivíduo poderia ter relação com a resposta ao TSH e que a dose mais aceitável seria de $14 \mathrm{ml} / \mathrm{kg}^{(12)}$.

O objetivo deste estudo é avaliar a influência do índice de massa corporal (IMC) e do peso corporal na flutuação e no pico da PIO no TSH no glaucoma primário de ângulo aberto (GPAA), glaucoma de pressão normal (GPN) e olhos normais (ON).

\section{Métodos}

Estudo transversal realizado no período de janeiro a dezembro de 2005, no Hospital Municipal da Piedade, Rio de Janeiro. Todos os pacientes assinaram na pri- meira consulta o termo de consentimento livre e esclarecido concordando em participar no estudo. O protocolo do estudo foi aprovado pelo Comitê de Ética do Hospital Municipal da Piedade.

Foram selecionados pacientes portadores de GPAA, GPN e indivíduos com ON, sendo divididos em três grupos: GPAA, GPN e ON.

Foram incluídos apenas indivíduos com idade acima de 35 anos e excluídos aqueles com cirurgia intraocular ou terapia a laser prévia, além de medicação oftalmológica ou sistêmica que interferissem na PIO. Nenhum dos portadores de glaucoma fazia uso de medicação antiglaucomatosa, pois o diagnóstico da doença foi realizado durante o estudo.

O IMC foi calculado pela forma padrão da razão entre peso do indivíduo em quilogramas e o quadrado de sua altura em metros ${ }^{(13)}$.

O TSH foi realizado sempre às 17 horas, com a ingestão de 1 litro de água pelo paciente em 5 minutos, sendo realizada a primeira tonometria após 15 minutos e, depois, medidas sucessivas com intervalos de 15 minutos em um total de quatro medidas. Todas as tonometrias foram realizadas com o mesmo tonômetro de aplanação de Goldmann e pelo mesmo examinador, após a verificação semanal da calibração do tonômetro. Os pacientes foram orientados a não ingerir alimentos ou líquidos após o almoço, às 12 horas, até o TSH.

Os pacientes portadores de GPAA e GPN apresentavam lesões características do nervo óptico ${ }^{(14)} \mathrm{e}$ defeitos correspondentes do campo visual ${ }^{(15)} \mathrm{com}$ diferenças dos níveis de PIO. Os pacientes com GPAA apresentavam pelo menos duas tonometrias acima de $21 \mathrm{mmHg}$ em pelo menos um olho, enquanto aqueles com GPN não apresentavam nenhuma aferição da PIO acima de $21 \mathrm{mmHg}$, inclusive em curva diária de PIO (CDPIO).

O pico de PIO foi considerado como a maior medida obtida em cada paciente durante oTSH.A flutuação da PIO foi considerada como a diferença entre a PIO basal, medida antes da ingestão de água, e a maior tonometria obtida durante o TSH em cada grupo. Para efeito do estudo, foi avaliado apenas o olho direito de cada paciente.

Foram utilizados os seguintes métodos estatísticos: análise de variância (ANOVA) - fator único com teste de Bonferroni e correlação linear de Pearson, através dos programas Bioestat 3.0 e Excel. Foi considerado significativo valor de $\mathrm{p}$ menor que 0,05 .

${ }^{1}$ Schmidt K. Untersuchungen über allgemeine und lokale Kapillar-Endothelstörungen bei Glaukoma Simplex. Apud ${ }^{(2)}$ 
Tabela 1

Média e desvio padrão da idade dos três grupos

\begin{tabular}{crr}
\hline Grupo & N & Idade (anos) \\
\hline GPAA & 32 & $58,9 \pm 13,6$ \\
GPN & 30 & $59,8 \pm 11,7$ \\
ON & 20 & $52,6 \pm 13,4$ \\
Valor de p & & 0,13 \\
\hline
\end{tabular}

GPAA - Glaucoma primário de ângulo aberto;

GPN - Glaucoma de pressão normal; ON - olhos normais.

Teste estatístico: análise de variância (ANOVA)

fator único. $\mathrm{p}=0.05$
Tabela 2

Média e desvio padrão do peso corporal e do índice de massa corporal dos três grupos

\begin{tabular}{cccc}
\hline Grupo & N & IMC & Peso Corporal \\
\hline GPAA & 32 & $28,4 \pm 4,8$ & $69,3 \pm 12,6$ \\
GPN & 30 & $28 \pm 4,7$ & $67,4 \pm 11,3$ \\
ON & 20 & $25,9 \pm 3,4$ & $68 \pm 10,8$ \\
Valor de p & & 0,13 & 0,83 \\
\hline
\end{tabular}

GPAA - Glaucoma primário de ângulo aberto;

GPN - Glaucoma de pressão normal; ON - olhos normais;

IMC - Índice de massa corporal. Teste estatístico: análise de variância (ANOVA) fator único. $\mathrm{p}=0.05$

Tabela 3

Correlação entre o índice de massa corporal, o peso corporal e a flutuação e os picos de pressão intra-ocular do teste de sobrecarga hídrica

\begin{tabular}{ccccccccc}
\hline & \multicolumn{2}{c}{ IMC x Flut } & \multicolumn{2}{c}{ IMC x Pico } & \multicolumn{2}{c}{ PC x Flut } & \multicolumn{2}{c}{ PC x Pico } \\
\hline & $\mathrm{R}$ & $\mathrm{p}$ & $\mathrm{R}$ & $\mathrm{p}$ & $\mathrm{R}$ & $\mathrm{p}$ & $\mathrm{R}$ & $\mathrm{p}$ \\
GPAA & $-0,41$ & $0,02^{*}$ & $-0,28$ & 0,11 & $-0,42$ & $0,02^{*}$ & $-0,2$ & 0,27 \\
GPN & $-0,22$ & 0,24 & $-0,07$ & 0,7 & $-0,06$ & 0,76 & $-0,13$ & 0,5 \\
ON & $-0,31$ & 0,19 & 0,14 & 0,56 & $-0,35$ & 0,13 & $-0,01$ & 0,95 \\
TODOS & $-0,21$ & 0,05 & 0,05 & 0,63 & $-0,23$ & $0,03^{*}$ & $-0,04$ & 0,72 \\
\hline
\end{tabular}

GPAA - Glaucoma primário de ângulo aberto; GPN - Glaucoma de pressão normal

ON - olhos normais; TODOS - Todos os grupos; IMC - Índice de massa corporal; PC - Peso corporal; Flut - flutuação. Teste estatístico: correlação linear de Pearson. $\mathrm{p}=0.05$. * = estatisticamente significativo

Tabela 4

Média dos picos de pressão intra-ocular nos três grupos

\begin{tabular}{lccc}
\hline & $\begin{array}{c}\text { GPAA } \\
\text { PIO }(\mathbf{m m H g})\end{array}$ & $\begin{array}{c}\text { GPNO } \\
(\mathbf{m m H g})\end{array}$ & $\begin{array}{c}\text { ON } \\
\text { PIO }(\mathbf{m m H g})\end{array}$ \\
\hline Valor de $\mathrm{p}$ & $26,8 \pm 4,3$ & $19,5 \pm 2,8$ & $16,1 \pm 2,6$ \\
Bonferroni: & $<0,01$ & & \\
GPAA e GPN & $\mathrm{p}$ & & \\
GPAA e ON & $<0,001$ & & \\
GPN e ON & $<0,001$ & & \\
\hline
\end{tabular}

GPAA - Glaucoma primário de ângulo aberto; GPN Glaucoma de pressão normal; ON - olhos normais; PIO - pressão intra-ocular. Teste estatístico: análise de variância (anova) fator único com teste de Bonferroni $\mathrm{p}=0.05$
Tabela 5

Média da flutuação da pressão intra-ocular nos três grupos

\begin{tabular}{lccc}
\hline & $\begin{array}{c}\text { GPAA } \\
\text { PIO }(\mathbf{m m H g})\end{array}$ & $\begin{array}{c}\text { GPNO } \\
(\mathbf{m m H g})\end{array}$ & $\begin{array}{c}\text { ONO } \\
(\mathbf{m m H g})\end{array}$ \\
\hline Valor de $\mathrm{p}$ & $3,97 \pm 1,9$ & $2,7 \pm 1,4$ & $2,8 \pm 0,4$ \\
Bonferroni: & $<0,01$ & & \\
GPAA e GPN & $\mathrm{p}$ & & \\
GPAA e ON & $<0,01$ & & \\
GPN e ON & $>0,03$ & & \\
\hline
\end{tabular}

GPAA - Glaucoma primário de ângulo aberto; GPN Glaucoma de pressão normal; ON - olhos normais; PIO - pressão intra-ocular. Teste estatístico: análise de variância (anova) fator único com teste de Bonferroni $\mathrm{p}=0.05$ 


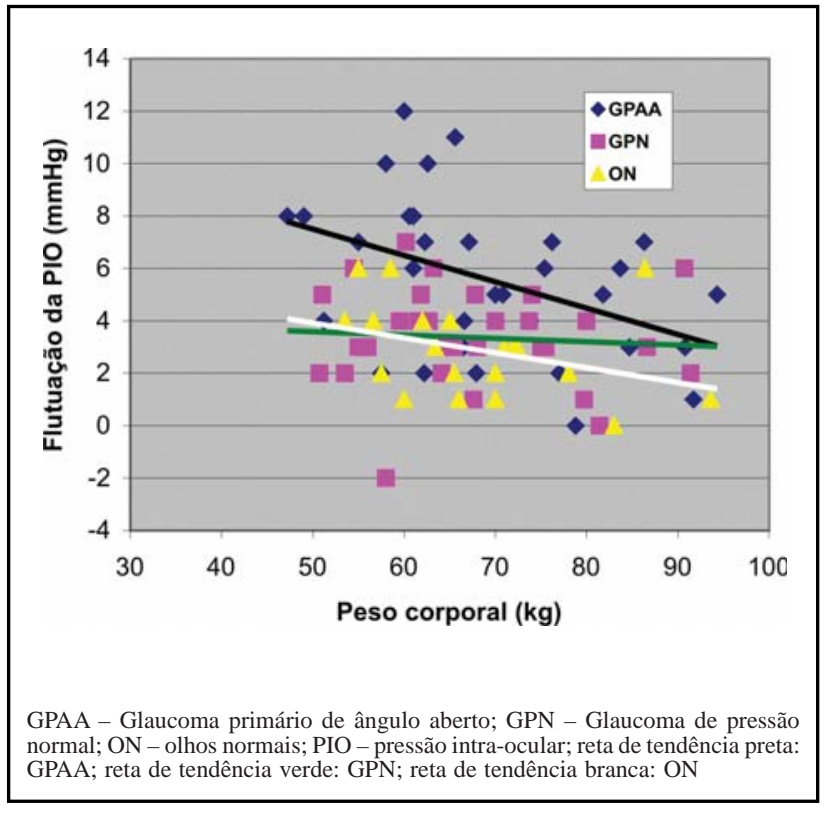

Figura 1: Correlação entre o peso corporal e a flutuação da PIO nos três grupos

\section{Resultados}

Foram incluídos no estudo 82 olhos de 82 pacientes, sendo divididos em três grupos: GPAA, GPN e ON. O grupo GPAA foi constituído de 32 pacientes, sendo 8 do sexo masculino e 24 do sexo feminino. O grupo GPN foi constituído de 30 pacientes, sendo 5 do sexo masculino e 25 do sexo feminino. $\mathrm{O}$ grupo $\mathrm{ON}$ foi composto por 20 pacientes, sendo 8 do sexo masculino e 12 do sexo feminino.

A Tabela 1 mostra que não houve diferença significativa da idade entre os três grupos, em ambos os olhos $(\mathrm{p}=0,13)$. Não houve, também, diferença significativa entre o IMC nos três grupos $(p=0,13)$ (Tabela 2$)$ e entre o peso corporal nos três grupos $(\mathrm{p}=0,83)$ (Tabela 2).

Houve correlação estatisticamente significativa entre a flutuação da PIO e o IMC ( $r=-0,41 ; p=0,02)$, entre a flutuação da PIO e o peso corporal $(r=-0,42$; $\mathrm{p}=0,02)$ no grupo GPAA e entre a flutuação da PIO e o peso corporal nos três grupos em conjunto (TODOS) ( $\mathrm{r}$ $=-0,23 ; p=0,03)$ (Tabela 3). Não houve correlação estatisticamente significativa entre os picos de PIO e o IMC ou o peso corporal em nenhum dos grupos, em separado ou em conjunto (Tabela 3). As Figuras 1 e 2 mostram, respectivamente, a dispersão em gráficos de correlação, entre a flutuação da PIO e o peso corporal no TSH e a flutuação da PIO e o IMC no TSH. Estes gráficos apresentam retas de tendência negativas nos três grupos, que é mais acentuada no grupo GPAA, no qual houve corre-

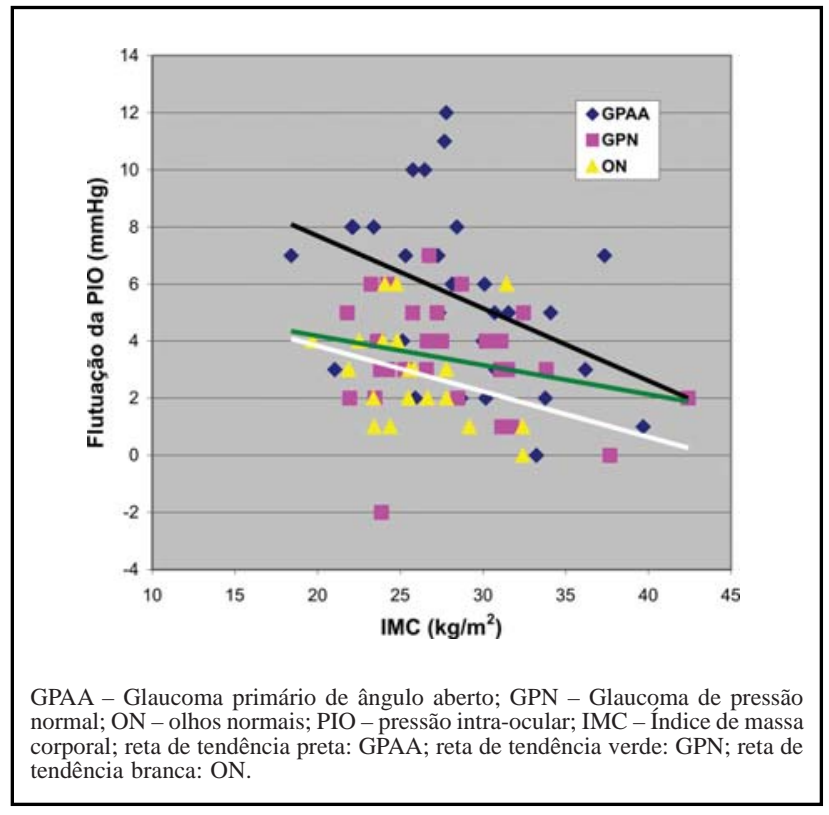

Figura 2: Correlação entre o IMC e a flutuação da PIO nos três grupos

lação significativa.

Os picos de PIO foram estatisticamente maiores no grupo GPAA e menores no grupo ON $(\mathrm{p}<0,001)$, como mostra a $T$ abela 4. A flutuação da PIO durante o TSH foi estatisticamente maior no grupo GPAA $(p<0,01)$ (Tabela 5). Não houve diferença estatisticamente significativa entre a flutuação do grupo GPN e a flutuação do grupo ON ( $>>0,05)$ (Tabela 5).

\section{Discussão}

Os grupos foram homogêneos em relação à idade, ao peso corporal e ao IMC, não havendo diferença estatisticamente significativa relacionada a estas variáveis entre os grupos. Os picos e a flutuação da PIO no TSH foram maiores no grupo GPAA. Foi encontrada correlação significativa entre o peso corporal, o IMC e a flutuação da PIO no grupo GPAA. As retas de tendência, nos gráficos de dispersão (Figuras 1 e 2), foram negativas no grupo GPAA, sugerindo que, quanto maior o peso corporal e o IMC, menor é a flutuação da PIO. Nos outros grupos , GPN e ON, esta correlação não foi estatisticamente significativa, embora as retas de tendência, destes grupos, também tenham sido negativas.

O mecanismo do aumento da PIO, após a ingestão de água no TSH, ainda está sujeito a controvérsias. Estudos iniciais associaram o aumento da PIO no TSH à diminuição da osmolaridade sanguínea ${ }^{(3,16-17)}$. Outros tra- 
balhos, entretanto, verificaram uma diminuição da facilidade de saída do humor aquoso, revelada pela tonografia, durante o $\mathrm{TSH}^{(18-19)}$. Outro estudo considerou que outros fatores, além da osmolaridade, como a pressão venosa, poderiam estar envolvidos no aumento da PIO, após o TSH ${ }^{(12)}$. Foi verificado ainda que após instilação de fluoresceína, seis horas antes da realização do TSH, havia um refluxo deste corante dez minutos após a ingestão de água, que seria causado pelo aumento da pressão venosa episcleral ${ }^{(11)}$. Outro trabalho ${ }^{(20)}$ não encontrou alterações da pressão osmótica ou do hematócrito, nem correlação destas variáveis ou da osmolaridade do plasma com o aumento da PIO durante o TSH. Os autores concluíram que fatores que alterariam a drenagem do humor aquoso poderiam explicar o efeito da ingestão da água.

Pouco se sabe sobre a influência do peso corporal e do IMC nos resultados do TSH. Um estudo anterior, ao pesquisar diversas variáveis relacionadas ao TSH, considerou que a dosagem ideal de água para ser ingerida por cada indivíduo no TSH seria de $14 \mathrm{ml} / \mathrm{kg}^{(12)}$. O fato de, no presente estudo, ter sido encontrada correlação significativa entre a flutuação da PIO e o peso corporal e o IMC apenas no grupo GPAA, poderia ser explicada pela maior flutuação da PIO encontrada nos portadores de GPAA. Outros estudos relataram, em curvas diárias de PIO, maior flutuação da PIO em pacientes com GPAA, comparados a indivíduos com $\mathrm{ON}^{(21)}$, e flutuações similares da PIO em portadores de GPN e indivíduos com ON ${ }^{(22)}$.

Alguns estudos sugerem que os picos e a flutuação da PIO durante o TSH seriam um bom indicativo do funcionamento do sistema de drenagem no trabeculado ${ }^{(7,10)}$. No presente estudo, foi encontrada correlação apenas entre a flutuação da PIO no TSH e o IMC e o peso corporal no grupo GPAA e não o entre o pico de PIO e o IMC ou o peso corporal. Isto sugere que o IMC e o peso corporal podem influenciar o quanto a PIO se eleva durante o TSH após a ingestão de água e não o pico da PIO no TSH isoladamente.

Alguns pacientes podem ter realizado o TSH no horário do seu pico diário de PIO e, com isso, apresentado uma pequena flutuação, influenciando os resultados do trabalho. Pacientes que realizam o TSH no horário do pico de PIO geralmente apresentam pequenas variações da PIO durante o TSH ${ }^{(5.6)}$. No entanto, realizamos o TSH em todos os pacientes às 17 horas, horário em que a PIO costuma, na maioria dos indivíduos, estar mais baixa ${ }^{(23-24)}$.

Ao contrário de estudos que encontraram maior pico e maior flutuação da PIO no TSH em pacientes sob tratamento antiglaucomatoso ${ }^{(7,1)}$ que apresentavam pro- gressão do glaucoma, o presente trabalho foi realizado em pacientes que ainda não estavam em uso de qualquer droga antiglaucomatosa, a qual poderia interferir nos resultados.

Solicitamos aos pacientes que não se alimentassem ou ingerissem água até 5 horas antes do TSH. Estudo anterior, no entanto, não encontrou influência da alimentação nos resultados do TSH ${ }^{(25)}$.

Este estudo se limitou a avaliar a influência do peso corporal e do IMC nos resultados do TSH, sem considerar outros fatores que poderiam influenciar nos resultados, como correlação do TSH com o pico diário de PIO ou a gravidade do glaucoma.

Apesar de a correlação entre o IMC, o peso corporal e a flutuação da PIO no TSH ter sido apenas razoável no grupo GPAA, esta associação sugere que, em alguns pacientes, estas variáveis podem influenciar nos resultados do TSH. Novos estudos devem ser realizados para se avaliar a quantidade de água que cada indivíduo deve ingerir para realizar o TSH, de acordo com o peso corporal e o IMC.

Os resultados do presente estudo sugerem que o peso corporal e o IMC devem ser considerados ao se avaliar os resultados do TSH em portadores de GPAA e que pacientes portadores de GPAA com maior IMC e maior peso corporal podem apresentar menor flutuação noTSH.

\section{Abstract}

Purpose: To evaluate the influence of the body weight and body mass index (BMI) on the intraocular pressure $(I O P)$ peaks and fluctuation in the water-drinking test $(W D T)$ in primary open angle (POAG) and normal tension glaucoma (NTG) patients, as well as normal eyes (NE). Methods: Thirty-two eyes of 32 POAG patients, 30 eyes of 30 NTG patients and 20 eyes of 20 normal patients were evaluated in this transversal study. None of the patients were using antiglaucomatous drugs at the beginning of the study. The WDT was performed at 5:00 pm for further correlation between body weight, BMI and intraocular pressure peaks and fluctuation. Analysis of variance, Bonferroni test and Pearson correlation coefficient were used for statistical analysis. Inherent to the methods used, $p$-values below 0,05 were considered to ensure statistical relevance. Results: There was no statically significant difference between age $(p=0,13)$, body weight $(p=0,13)$ and BMI $(0,83)$ in the three groups studied. There was significant correlation between body weight and IOP fluctuations $(p=0,02 ; r=-0,42)$ and between BMI and IOP fluctuations $(p=0,02 ; r=-0,41)$ in 
POAG patients. Body weight and BMI were not significantly associated with IOP peaks in the three groups. There was no statistically significant correlation between body weight, BMI and IOP fluctuations in NTG patients and in NE. Conclusion: The results suggest that knowledge of body weight and BMI is advisable before interpreting the WDT outcomes in POAG patients. POAG patients with higher body weight and BMI may present lower fluctuation in the WDT.

Keywords: Open-angle glaucoma/diagnosis; Intraocular pressure; Diagnostic techniques, ophthalmological; Ocular tonometry/methods; Body weight; Body mass index; Case control studies

\section{REFERÊNCIAS}

1. Zeimer RC, Wilensky JT, Gieser DK. Presence and rapid decline of early morning intraocular pressure peaks in glaucoma patients. Ophthalmology. 1990; 97(5): 547-50.

2. Schmidt K. Untersuchungen über allgemeine und lokale Kapillar-Endothelstörungen bei Glaukoma Simplex. Apud Sugar HS. The provocative tests in the diagnosis of the glaucomas. Am J Ophthalmol. 1948; 31(10): 1193-210.

3. Leydhecker W . The water-drinking test. Br J Ophthalmol. 1950; 34(8): 457-79.

4. Susanna Júnior R, Campagna CM. Sobrecarga hídrica: sua importância no diagnóstico do glaucoma. Rev Bras Oftalmol. 1988; 47(3): 157-8.

5. Miller D. The relationship between diurnal tension variation and the water-drinking test. Am J Ophthalmol. 1964; 58: 243-6.

6. Helal Júnior J. Contribuição ao estudo da pressão intra-ocular: picos de pressão intra-ocular na curva diária de pressão e na prova de sobrecarga hídrica. Rev Bras Oftalmol. 1988; 47(2): 75-80.

7. Susanna R Jr, Vessani RM, Sakata L, Zacarias LC, Hatanaka $M$. The relation between intraocular pressure peak in the water drinking test and visual field progression in glaucoma. Br J Ophthalmol. 2005; 89(10): 1298-301.

8. Medeiros FA, Pinheiro A, Moura FC, Leal BC, Susanna R Jr. Intraocular pressure fluctuations in medical versus surgically treated glaucomatous patients. J Ocul Pharmacol Ther. 2002; 18(6): 489-98.

9. Chen $\mathrm{CH}, \mathrm{Lu}$ DW, Chang CJ, Chiang $\mathrm{CH}$, Chou PI. The application of water drinking test on the evaluation of trabeculectomy patency. J Ocul Pharmacol Ther. 2000; 16(1): $37-42$.
10. Malerbi FK, Hatanaka M, Vessani RM, Susanna R Jr. Intraocular pressure variability in patients who reached target intraocular pressure. Br J Ophthalmol. 2005; 89(5):540-2.

11. Diestellhorst M, Krieglstein GK. The effect of water-drinking test on aqueous humor dynamics in healthy volunteers. Graefes Arch Clin Exp Ophthalmol. 1994; 232(3): 145-7.

12. Spaeth GL. The water drinking test. Indications that factors other than osmotic considerations are involved. Arch Ophthalmol. 1967; 77(1): 50-8.

13. Atkinson RL. Guidelines for the initiation of obesity treatment. J Nutr Biochem. 1998; 9(10):546-52.

14. Jonas JB, Budde WM, Panda-Jonas S. Ophthalmoscopic evaluation of the optic nerve head. Surv Ophthalmol. 1999; 43(4): 293-320. Review.

15. Comparison of glaucomatous progression between untreated patients with normal-tension glaucoma and patients with therapeutically reduced intraocular pressures. Collaborative Normal-Tension Glaucoma Study Group. Am J Ophthalmol. 1998; 126(4): 487-97. Erratum in: Am J Ophthalmol. 1999;127(1):120.

16. Campbell DA, Gloster J, Tonks EL. Some observations on the water-drinking test in glaucomatous and non-glaucomatous subjects. Br J Ophthalmol. 1955; 39(4): 193-203.

17. Galin MA. Effect of the water provocative test on ocular rigidity. Arch Ophthalmol. 1963; 70: 337-41.

18. Becker B, Christensen RE. Waterdrinking and tonography in the diagnosis of glaucoma. AMA Arch Ophthalmol. 1956; 56(3): 321-6.

19. Armaly MF, Sayegh RE. Water-drinking-test. II. The effect of age on tonometric and tonographic measures. Arch Ophthalmol. 1970; 83(2): 176-81.

20. Brucculeri M, Hammel T, Harris A, Malinovsky V, Martin B. Regulation of intraocular pressure after water drinking. J Glaucoma. 1999; 8(2): 111-6.

21. Drance SM. The significance of the diurnal tension variations in normal and glaucomatous eyes. Arch Ophthalmol. 1960; 64: 494-501.

22. De Vivero C, O'Brien C, Lanigan L, Hitchings R. Diurnal intraocular pressure variation in low-tension glaucoma. Eye. 1994; 8(Pt 5): 521-3.

23. Liu JH, Kripke DF, Hoffman RE, Twa MD, Loving RT, Rex $\mathrm{KM}$, et al. Elevation of human intraocular pressure at night under moderate illumination. Invest Ophthalmol Vis Sci. 1999; 40(10): 2439-42.

24. Meirelles SHS, Yamane R, Álvares RM, Botelho PB, Morais FB, Moreira PB, et al. Estudo comparativo entre a curva diária de pressão intra-ocular e a associação da curva ambulatorial com o teste de sobrecarga hídrica no glaucoma primário de ângulo aberto, glaucoma de pressão normal e olhos normais. Arq Bras Oftalmol. 2007; 70(3): 471-9.

25. Mehra KS. Water drinking provocative test. Ann Ophthalmol. 1979; 11(2): 223-4. 